\title{
A two-stage review process for randomized controlled trials: the ultimate solution for publication bias?
}

\author{
Lawrence Siu-Chun Law, MD (1) - Elaine Ah-Gi Lo, PharmD, BCPS
}

Received: 27 July 2016/Revised: 8 August 2016/ Accepted: 17 August 2016/Published online: 13 September 2016

(c) Canadian Anesthesiologists' Society 2016

\section{To the Editor,}

We read with interest the study published by Chong et al. ${ }^{1}$ The authors traced 1,052 abstracts of randomized, controlled studies presented at the 2001-2004 American Society of Anesthesiologists' annual meetings. Their results showed that only $53.6 \%$ of the abstracts were published during the subsequent ten years. Also, the abstracts with positive results were more likely to proceed to publication than those with negative results (relative risk 1.42, $P<$ 0.001 ), implying potential publication bias in the anesthesia literature for studies with positive outcomes.

Indeed, publication bias is not only limited to the field of anesthesia, it is also common in other disciplines. ${ }^{2}$ Current efforts to remedy this problem have included the following: (1) allowing only registered trials to be published; (2) the existence of open access journals that emphasize methodology rather than results in the review process; (3) encouragement from major journals to publish studies with

This letter is accompanied by a reply. Please see Can J Anesth 2016; 63: this issue.

L. S.-C. Law, MD

Department of Anaesthesia, National University Hospital,

Singapore, Singapore

L. S.-C. Law, MD ( $\square)$

Center for Quantitative Medicine, Duke-NUS Graduate Medical

School, Singapore, Singapore

e-mail: sclaw@alumni.duke.edu

E. A.-G. Lo, PharmD, BCPS

Department of Pharmacy, National University Hospital,

Singapore, Singapore

E. A.-G. Lo, PharmD, BCPS

Department of Pharmacy, The University of Hong Kong, Pok Fu Lam, Hong Kong SAR rigorous methodology on important research topics despite negative outcomes. By registering all trials, the scientific communities are able to acknowledge the existence of unpublished trials and take those trials into account for meta-analyses, thereby reducing the effect of publication bias. Focusing on methodology and de-emphasizing results in open access and major journals may increase the chance of publishing negative studies with good methodology. These solutions, however, do not completely resolve all sources of publication bias. ${ }^{2}$

The editorial by Jones ${ }^{3}$ suggested several solutions for reducing publication bias further, including additional research focused on publication bias, an emphasis by university and funding bodies on the quality of the study rather than the results alone, and having research ethics boards make it compulsory to publish all registered studies regardless of outcomes.

In addition to these suggestions, we propose a two-stage review process to minimize the influence of results during review. ${ }^{4,5}$ In the first stage, editors and reviewers would be blinded to the results and parts of the discussion that refer to the results. Authors would submit the manuscript with an additional discussion about the potential implication of both positive and negative results for clinical practice and future research. The decision of provisional acceptance would be based purely on the background, methodology, baseline characteristics of participants, and potential implications. If the study was then considered suitable for publication, the manuscript would enter the second stage of review, during which the entire manuscript would be considered in the usual manner.

The potential benefits would be the following:

1. Reviewers and editors are blinded to the negative results, thereby eliminating the biases for positive outcomes. 
2. Rejecting studies with positive results but poor methodology, which is as important as accepting studies with negative results but good methodology.

3. Authors' confidence to submit good studies with negative outcomes is enhanced as bias may originate from authors' decision to hold back manuscripts with negative results.

This proposal is compatible with the existing framework of peer-reviewing process, reducing the effect of publication bias on future meta-analyses.

The potential drawbacks would be the following:

1. It is inconvenient, as the proposal would increase the reviewing process workload.

2. Journals would likely publish more papers that were previously considered "non-publishable" (i.e., negative), which might affect the journal's impact factor.

3. Authors may not be able to provide results and discussion that are up to publishable standards after acceptance following the first stage of the review. We believe, however, that the chance of having substandard results and discussion is relatively low for studies with a good statistical plan, an adequate size based on a priori power analysis, unbiased sampling as shown by the baseline characteristics, and significant clinical and research implications for positive and/or negative results.
For the sake of science and truth, we believe that the benefits would outweigh the drawbacks, and implementing the proposed two-stage review process would reduce publication bias for randomized controlled trials.

Funding None

Conflict of interest Authors declare no conflict of interest.

Editorial responsibility This submission was handled by Dr. Hilary P. Grocott, Editor-in-Chief, Canadian Journal of Anesthesia.

\section{References}

1. Chong SW, Collins NF, Wu CY, Liskaser GM, Peyton PJ. The relationship between study findings and publication outcome in anesthesia research: a retrospective observational study examining publication bias. Can J Anesth 2016; 63: 682-90.

2. Kicinski M, Springate DA, Kontopantelis E. Publication bias in meta-analyses from the Cochrane Database of Systematic Reviews. Stat Med 2015; 34: 2781-93.

3. Jones PM. Publication bias in the anesthesiology literature: shifting the focus from the "positive" to the "truth". Can J Anesth 2016; 63: 658-63.

4. Mell LK, Zietman $A L$. Introducing prospective manuscript review to address publication bias. Int J Radiat Oncol Biol Phys 2014; 90: 729-32.

5. Chambers $C D$. Registered reports: A new publishing initiative at Cortex. Cortex 2013; 49: 609-10. 